\title{
Activité antidrépanocytaire et thermodégradation des anthocyanes extraits de Sterculia quinqueloba et Ficus capensis
}

\author{
P. T. MPIANA ${ }^{1}{ }^{*}$, E. K. BALANGANAYI ${ }^{2}$, A. B. KANANGILA ${ }^{2}$, E. M. KALONDA ${ }^{2}$, \\ K. N. NGBOLUA ${ }^{3}$, D. S. T. TSHIBANGU ${ }^{1}$, E. K. ATIBU ${ }^{1}$ et J. B. S. LUMBU $^{2}$ \\ ${ }^{1}$ Département de Chimie, Faculté des Sciences B.P. 190, Université de Kinshasa, Kinshasa XI, R. D. Congo. \\ ${ }^{2}$ Département de Chimie, Faculté des Sciences, Université de Lubumbashi, Lubumbashi, R. D. Congo. \\ ${ }^{3}$ Département de Biologie, Faculté des Sciences B.P. 190, Université de Kinshasa, Kinshasa XI, R. D. Congo. \\ *Auteur correspondant, E-mail : ptmpiana@yahoo.fr; Tel: (+243) 818116019
}

\begin{abstract}
ASBSTRACT
L'activité antifalcémiante de Ficus capensis et Sterculia quinqueloba, deux plantes utilisées en médicine traditionnelle contre la drépanocytose dans la ville de Lubumbashi (RD Congo), a été testée en utilisant le test d'Emmel. Les résultats obtenus ont montré une activité antifalcémiante importante in vitro pour les deux plantes. Cette activité serait due aux anthocyanes. Les concentrations minimales de normalisation des drépanocytes obtenus sont de 2,5 $\mu \mathrm{g} / \mathrm{ml}$ et $3,0 \mu \mathrm{g} / \mathrm{ml}$ pour les anthocyanes de Ficus capensis et de Sterculia quinqueloba respectivement. Le fractionnement de ces anthocyanes par chromatographie sur couche mince utilisant les mélanges des solvants acétate d'éthyle-éthanol 96\%-eau distillée-acide acétique $10: 5: 10: 1$ pour Ficus capensis et benzène-méthanol-éthanol 96\%-acide acétique $2: 4: 1: 1$ a montré que ce sont les fractions ayant respectivement les rapports frontaux de 0,49 et 0,66 qui sont les plus actives. Ces anthocyanes ont montré une instabilité vis-à-vis de la chaleur. La cinétique de thermodégradation de la fraction la plus active des anthocyanes de Ficus capensis a donné les valeurs des constantes de vitesse expérimentales de $3,410^{-4} \mathrm{~s}^{-1}$ à $363 \mathrm{~K}$ et de $6,210^{-4} \mathrm{~s}^{-1}$ à $393 \mathrm{~K}$.

(c) 2009 International Formulae Group. All rights reserved.
\end{abstract}

Keywords : Drépanocytose, Test d'Emmel, Concentration Minimale de Normalisation, Taux de normalisation.

\section{INTRODUCTION}

La drépanocytose ou anémie falciforme est une maladie génétique qui frappe particulièrement les régions tropicales. C'est une hémoglobinopathie due au remplacement de l'acide glutamique situé en sixième position de la chaîne $\beta$ de l'hémoglobine $(\mathrm{Hb})$ par la valine. Cette substitution d'acides aminés modifie non seulement l'affinité de l'hémoglobine pour l'oxygène mais aussi sa solubilité dans les conditions de faible pression en oxygène. La baisse de la solubilité entraîne la polymérisation de l'hémoglobine et la falciformation des globules rouges. Ce qui est à la base de nombreux maux dont souffrent les sujets anémiques SS (Kaplan et Depech, 1993; Voet et Voet, 1998).

La drépanocytose affecte chaque année près de 5 millions de personnes dans le monde. Dans certaines régions d'Afrique, les porteurs du trait drépanocytaire représentent jusqu'à $20 \%$ de la population avec une prévalence en Afrique Centrale de 25 à $30 \%$ (Voet et Voet, 1998). Deux pourcent de la population en République Démocratique du Congo (RDC) sont touchés par cette maladie, soit plus d'un million d'habitants (Mpiana et al., 2007a). Près de $80 \%$ d'enfants atteints par cette anémie meurent avant leur cinquième anniversaire s'ils ne sont pas suivis 
médicalement (Stryer et al., 1997; Voet et Voet, 1998)

Plusieurs modes de traitements ont été envisagés en vue de soulager les malades, entre autre le greffage de la moelle osseuse, la thérapie génétique, les transfusions sanguines répétées, la prise de l'hydroxyurée etc., Mais il s'avère que ces traitements sont non seulement inefficaces et très coûteux pour les populations pauvres d'Afrique, mais peuvent constituer également un risque d'infection au VIH/SIDA (Sofowora, 1998; Wajcman, 2003; Eliot et al., 2006).

Ces dernières années, plusieurs études se sont tournées vers l'utilisation des plantes médicinales pour soigner la drépanocytose (Neuwinger, 2000; Iyamu et al., 2002; Moody et al., 2003; Ibrahim et al., 2007). Notre équipe de recherche a montré que plusieurs plantes médicinales utilisées en médecine traditionnelle congolaise pour soigner l'anémie SS avaient, in vitro, une activité antifalcémiante c'est-à-dire qu'elles empêchaient la falciformation des érythrocytes du sang SS (Mpiana et al., 2007a ; Mpiana et al., 2008a). Nos résultats antérieurs ont en outre montré que cette activité antifalcémiante était essentiellement due aux anthocyanes (Mpiana et al., 2007b; 2007c; 2007d; Mpiana et al., 2008a; 2008b ; 2008c; Mpiana et al., 2009).

En effet, les anthocyanes sont aujourd'hui en train de révéler plusieurs propriétés intéressantes, dont la capacité de lutter contre le vieillissement des tissus à cause de leur pouvoir antioxydant, l'activité contre le cancer etc. Mais ces pigments naturelles sont malheureusement instables vis-à-vis de certains paramètres physicochimiques tels que la chaleur, le $\mathrm{pH}$ et la lumière (Kahkoonen et al., 2003; Mpiana et al., 2008a ; Mpiana et al., 2009).

Dans cette étude, deux plantes, Ficus capensis et Sterculia quinqueloba, utilisées par les tradipraticiens de la contrée de Lubumbashi (RDC) pour soigner l'anémie SS, font l'objet de notre étude. Etant donné que la décoction est souvent recommandée comme mode de préparation des recettes traditionnelles, il est nécessaire non seulement de vérifier l'activité antifalcémiante de ces deux plantes, mais également de déterminer si ce sont les anthocyanes qui sont à la base de cette activité et d'étudier leur stabilité vis-àvis de la chaleur.

\section{MATERIEL ET METHODES Matériel végétal}

Les plantes ont été récoltées (feuilles et écorces) dans les environs de la ville de Lubumbashi au mois de juillet 2008. Les plantes récoltées ont été identifiées et déposées à l'Herbarium de la Faculté des Sciences de l'Université de Lubumbashi avec le numéro herbier 944 et 952.

\section{Extraction et test d'anthocyanes}

Les écorces des tiges et des racines du matériel végétal utilisé (100 g), séchées et pulvérisées à l'ombre, ont été macérées à trois reprises dans l'hexane, le dichlorométhane, le méthanol et l'eau (200 $\mathrm{ml} \mathrm{x} \mathrm{1)} \mathrm{pendant} 48$ heures. Les différentes fractions ont été filtrées et le test des anthocyanes réalisé sur chaque fraction en utilisant le mode opératoire universellement connu (Bruneton, 1999). L'extraction des anthocyanes a été réalisée par l'addition de l'éther diéthylique pour précipiter les anthocyanes dans le filtrat aqueux préalablement concentré à l'aide d'un évaporateur rotatif. Les anthocyanes ont été en suite récupérés et séchés tel que décrit précédemment (Mpiana et al., 2007b; Mpiana et al., 2008a).

\section{Fractionnement des anthocyanes}

La chromatographie sur couche mince a été réalisée sur les anthocyanes extraits du Ficus capensis avec comme système d'éluant le mélange acétate d'éthyle- éthanol 96\%-eau distillée - acide acétique anhydre dans les proportions $10: 5: 10: 1$ tandis que pour Sterculia quinqueloba, l'éluant été composé de benzène-méthanol-ethanol $96 \%$ acide acétique dans les proportions $2: 4: 1: 1$. Le fractionnement a été réalisé en utilisant une colonne chromatographique avec les mêmes systèmes d'éluants.

\section{Matériel biologique}

L'échantillon de sang hépariné utilisé pour évaluer l'activité antifalcémiante des plantes a été obtenu auprès de 12 adolescents affectés par la drépanocytose de l'«Association DREPANOCYTAIRESPARENTS-AMIS » au Centre Médical «MERE TERESSA » situé dans la commune 
de Kampemba à Lubumbashi (RDC). Le don de sang a été effectué avec le consentement éclairé des parents et des enfants. Aucun de ces patients n'a été récemment transfusé. Toutes les expériences sur l'activité antifalcémiante ont été réalisées avec un échantillon de sang fraîchement prélevé. Dans le but de confirmer leur nature SS, les échantillons de sang susmentionné ont été premièrement caractérisés par électrophorèse sur papier en utilisant le gel d'acétate de cellulose à $\mathrm{pH} 8,5$. Une fois la nature $\mathrm{SS}$ de l'échantillon de sang confirmée, ce dernier a été gardé à $\pm 4{ }^{\circ} \mathrm{C}$ dans un réfrigérateur.

\section{Activité antifalcémiante}

L'échantillon de sang a été mélangé avec les extraits des plantes à différentes concentrations (avec comme solvant de dissolution l'eau physiologique). Le test d'Emmel a été utilisé pour évaluer l'activité antifalcémiante (Courtejoie et Hartaing, 1992). Dans cette étude, le test d'Emmel a été modifié comme précédemment rapporté (Mpiana et al., 2007a). Les images microscopiques ont été obtenues en utilisant un microscope optique de marque MOTIC 30207598. Un appareil photographique KODAK EASY SHARE C613 a été utilisé en vue de numériser les micrographies. Ces dernières ont été ensuite traitées en utilisant le logiciel informatique MOTIC images 2000, version 1.3.

\section{Effet de la chaleur}

La thermodégradation a été réalisée en utilisant une étuve (MEMMERT type DEPEXBV) à différentes températures et à des temps d'exposition différents. Un spectrophotomètre UV-Visible de marque HACH DR/4000 U a été utilisé pour la lecture des densités optiques des solutions.

\footnotetext{
Modèle mathématique et analyse des données

La dégradation thermique des anthocyanes est considérée comme une réaction chimique. Ainsi, une molécule d'anthocyanes A est décomposée irréversiblement en une ou plusieurs molécules désignées par B. Cette transformation peut être représentée par l'équation suivante :
}

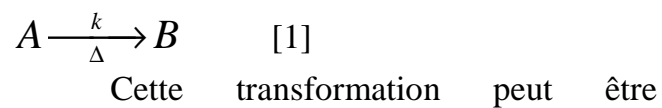
assimilée à une réaction de premier ordre pour laquelle l'équation est ci-dessous donnée: $\frac{d C_{A}}{d t}=-k C_{A}$

[2], Où $\mathrm{C}_{\mathrm{A}}$ et $\mathrm{t}$ sont

respectivement: la concentration de $\mathrm{A}$ et le temps de dégradation.

L'intégration de l'équation [2] donne: $C_{A}=C_{A}^{o} e^{-k t} \quad$ [3], Où $\mathrm{C}_{\mathrm{A}}{ }^{0}$ est la concentration initiale de $\mathrm{A}$.

Si A est le seul composé qui absorbe à la longueur d'onde choisie, la relation de Lamber-Beer dans ce cas devrait être : $E=l \varepsilon C_{A} \quad$ [4] et $E_{o}=l \varepsilon C_{A}^{o} \quad$ [5], où $\mathrm{E}, \mathrm{E}_{0}, 1$ et $\varepsilon$ sont respectivement l'absorbance au temps $t$, l'absorbance au temps, $\mathrm{t}=0$ seconde, le chemin optique et le coefficient d'extinction molaire.

La combinaison des équations [3], [4] et [5] donne $E=E_{o} e^{-k t} \quad$ [6].

$\mathrm{Si}$ le(s) composé(s) résultant du processus de dégradation (B) absorbe(nt) simultanément avec A à la même longueur d'onde, l'équation de Lamber-Beer pourrait devenir: $E=l\left(C_{A} \varepsilon_{A}+C_{B} \varepsilon_{B}\right)$ [7], où $\varepsilon_{\mathrm{A}}$, $\varepsilon_{\mathrm{B}}, \mathrm{C}_{\mathrm{A}}$ et $\mathrm{C}_{\mathrm{B}}$ sont respectivement les coefficients d'extinction molaire et les concentrations de A et B.

Considérant que: $C_{A}^{o}=C_{A}+C_{B}$ [8]. Et en combinant les équations [3] [5] [7] et [8], il vient que: $E=E_{\infty}+\frac{\varepsilon_{A}+\varepsilon_{B}}{\varepsilon_{A}} E_{o} e^{-k t} \quad$ [9], où $E_{\infty}=l \varepsilon_{A} C_{A}^{o}$

Si $\varepsilon_{\mathrm{A}}>\varepsilon_{\mathrm{B}}$ l'équation [9] donne la même allure exponentielle que l'équation [6].

En utilisant les deux modèles des équations [6] et [9], les résultats expérimentaux obtenus ont été traités par le logiciel informatique Microcal Origin 6.1.

\section{RESULTATS}

Quatre extraits ont été réalisés sur la poudre de deux plantes, il s'agit des extraits hexanique, dichlorométhanique, méthanolique et aqueux. Le test d'Emmel réalisé avec les différents extraits a montré une activité 
antifalcémiante élevée pour les extraits aqueux et méthanoliques (Tableau 1). Les figures 1,2 et 3 illustrent respectivement les micrographies du sang SS non traité (témoin), du sang SS traité avec les extraits aqueux de Ficus capensis et de Sterculia quinqueloba.

Comme on peut le remarquer, la figure 1 montre que la majorité des érythrocytes sont falciformes, ce qui confirme que notre échantillon est bel et bien un sang SS. Mais en présence des extraits aqueux de deux plantes, les drépanocytes reprennent dans leur majorité, la forme normale biconcave.

Le test des anthocyanes réalisé sur les différents extraits s'est avéré négatif sur les extraits hexaniques et dichlorométhaniques, mais positif sur les extraits aqueux et méthanoliques. Les figures 4 et 5 montrent les micrographies du sang SS en présence des extraits d'anthocyanes de Ficus capensis et Sterculia quinqueloba. Ces figures montrent que les drépanocytes reprennent la forme normale en présence des anthocyanes extraits de deux plantes.

Le taux de normalisation des drépanocytes en fonction de la concentration en anthocyanes de deux plantes évolue comme le montre la figure 6. Ce taux augmente au fur et à mesure que la concentration augmente pour atteindre une valeur maximale et constante.

Les anthocyanes extraits ont été ensuite fractionnés et testés en vue de déterminer les fractions les plus actives. L'extrait des

Tableau 1 : Taux de normalisation des drépanocytes par les différents extraits.

\begin{tabular}{llc}
\hline Plante & Type d'extraits & Taux de normalisation \\
\hline \multirow{3}{*}{ Ficus capensis } & Extraits bruts hexaniques & $12,5 \%$ \\
& Extraits bruts dichlorométhaniques & $16,5 \%$ \\
& Extraits bruts méthanoliques & $82,4 \%$ \\
& Extraits bruts aqueux & $89,7 \%$ \\
\hline \multirow{3}{*}{ Starculia quinqueloba } & Extraits bruts hexaniques & $4,8 \%$ \\
& Extraits bruts dichlorométhaniques & $19,7 \%$ \\
& Extraits bruts méthanoliques & $77,5 \%$ \\
& Extraits bruts aqueux & $83,3 \%$ \\
\hline
\end{tabular}

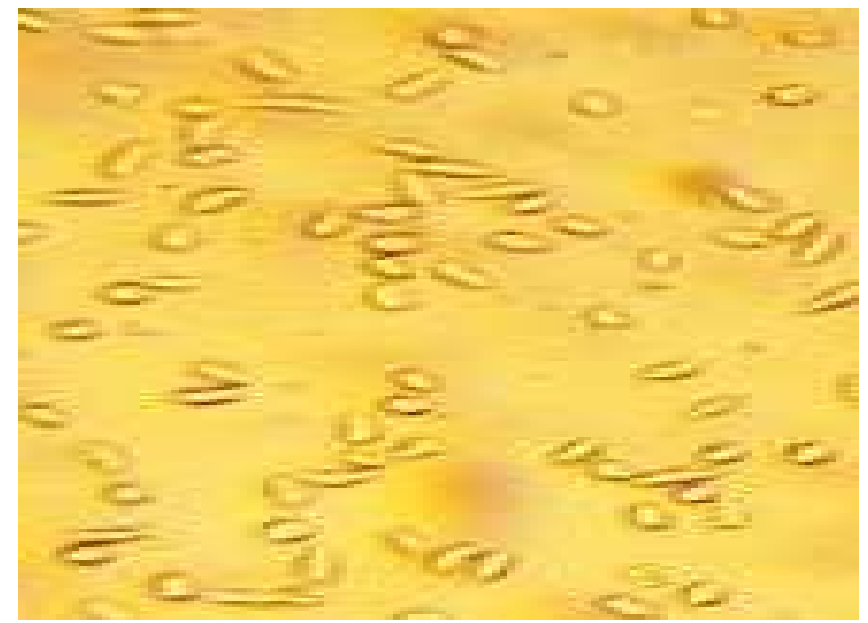

Figure 1: Morphologie des drépanocytes du sang SS non traité (500X). 


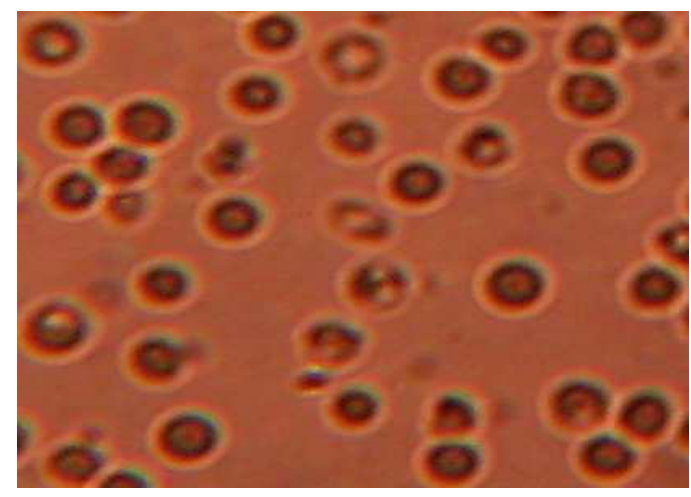

Figure 2: Morphologie des drépanocytes du sang SS traité avec les extraits aqueux de $F$. capensis (500X).

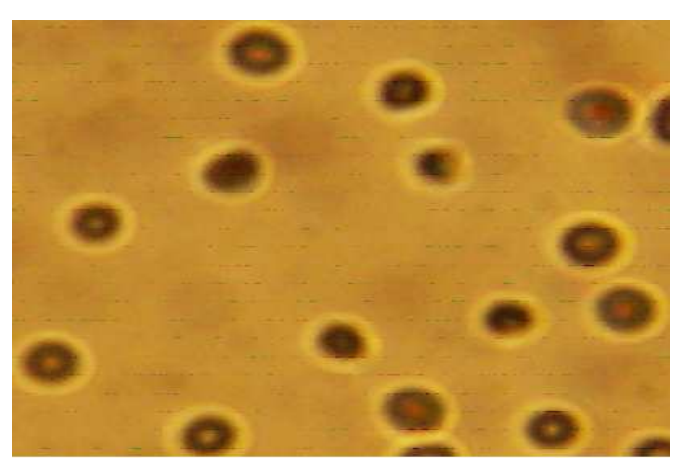

Figure 4: Morphologie des drépanocytes du sang SS traité avec les extraits totaux des anthocyanes de F. capensis (500X).

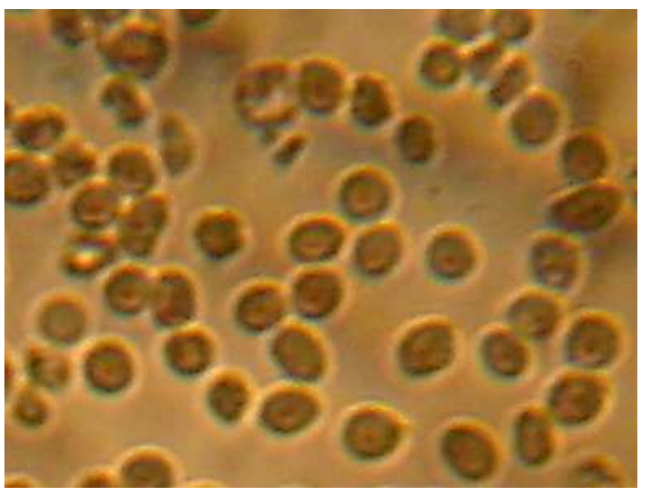

Figure 3: Morphologie des drépanocytes du sang SS traité avec les extraits aqueux de S. quinqueloba (500X).

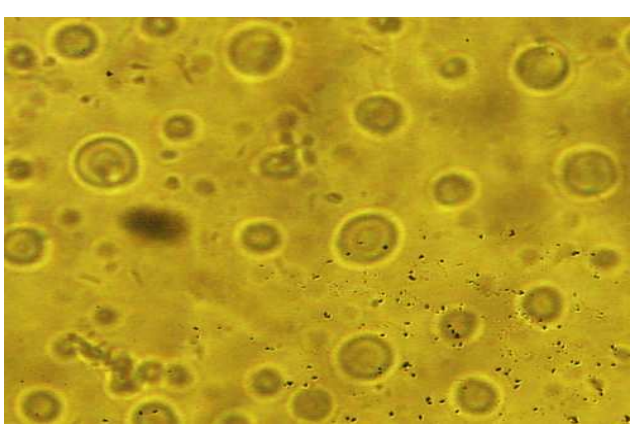

Figure 5: Morphologie des drépanocytes du sang SS traité avec les extraits totaux des anthocyanes de S .quinqueloba (500X).

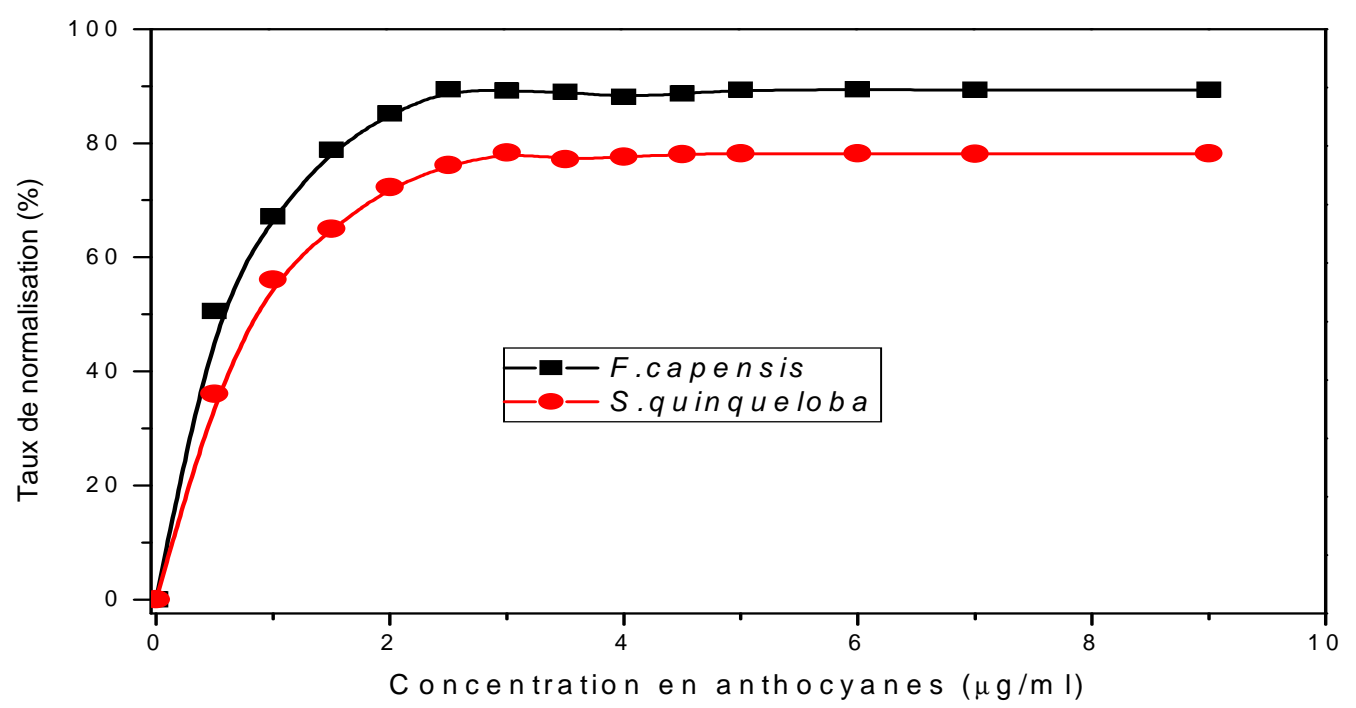

Figure 6: Taux de normalisation des drépanocytes en présence des extraits totaux d'anthocyanes de $F$. capensis et de $S$.quinqueloba. 
anthocyanes de Ficus capensis a donné trois fractions de rapports frontaux (Rf) suivants $\mathrm{Rf}_{1}=0,80, \mathrm{Rf}_{2}=0,49$ et $\mathrm{Rf}_{3}=0,30$ tandis celui de Sterculia quinqueloba a donné deux fractions de $\mathrm{Rf}_{1}=0,66$ et $\mathrm{Rf}_{2}=0,20$. Pour Ficus capensis c'est la fraction $2\left(\mathrm{~F}_{2}\right)\left(\mathrm{Rf}_{2}=0,49\right)$ qui a montré la plus grande activité tandis que pour Sterculia quinqueloba, c'est la fraction 1 $\left(\mathrm{S}_{1}\right)\left(\mathrm{Rf}_{1}=0,66\right)$ qui est la plus active. Le calcul fait sur plusieurs champs microscopiques a donné pour la fraction $\mathrm{F}_{2}$ un taux de normalisation atteignant $92 \%$ tandis que pour la fraction $\mathrm{S}_{1}$ il atteint $86 \%$.

Les spectres des fractions $F_{2}$ et $S_{1}$ dans le domaine de l'UV ont montrés chacun une bande autour de $270 \mathrm{~nm}$. Lorsque la fraction $\mathrm{F}_{2}$ a été soumise à la chaleur, cette bande a montré une décroissance pour les températures de chauffage croissantes à un temps de chauffage fixe et pour les temps d'exposition à la chaleur croissants à une température d'exposition fixe, comme l'illustrent les figures 7 et 8 .

La cinétique de thermodégradation de la fraction $F_{2}$ a alors été étudiée à 363 et 393 $\mathrm{K}$ en suivant l'évolution de la densité optique à $275 \mathrm{~nm}$ en fonction du temps de chauffage. Les points expérimentaux sont compatibles avec la courbe calculée selon l'équation [6] comme l'illustre la figure 9. Les valeurs des constantes de vitesse de dégradation thermique calculées sont de $3,410^{-4} \mathrm{~s}^{-1}$ et 6,2 $10^{-4} \mathrm{~s}^{-1}$ respectivement à $363 \mathrm{~K}$ et à $393 \mathrm{~K}$.

\section{DISCUSSION}

$\mathrm{Si}$ on compare les figures 2 et 3 avec la figure 1 , on peut dire que les extraits aqueux de $F$. capensis et de $S$. quinqueloba ont la capacité de remettre les érythrocytes falciformes dans leur forme normale parce que toutes ces micrographies ont été réalisées en conditions hypoxiques. Ce qui indique que ces deux plantes ont une activité antifalcémiante. Ces résultats confirment, en ce qui concerne $F$. capensis, nos résultats antérieurs (Mpiana et al., 2008a). Ils justifient donc l'utilisation de ces plantes en médecine traditionnelle contre la drépanocytose.

Pour pouvoir déterminer le groupe chimique responsable de cette activité, nous avons faits les extraits dans quatre solvants de polarités croissantes à savoir l'hexane, le dichlorométhane, le méthanol et l'eau. Le taux de normalisation des drépanocytes calculé pour les différents extraits (Tableau 1) a montré que l'activité antifalcémiante

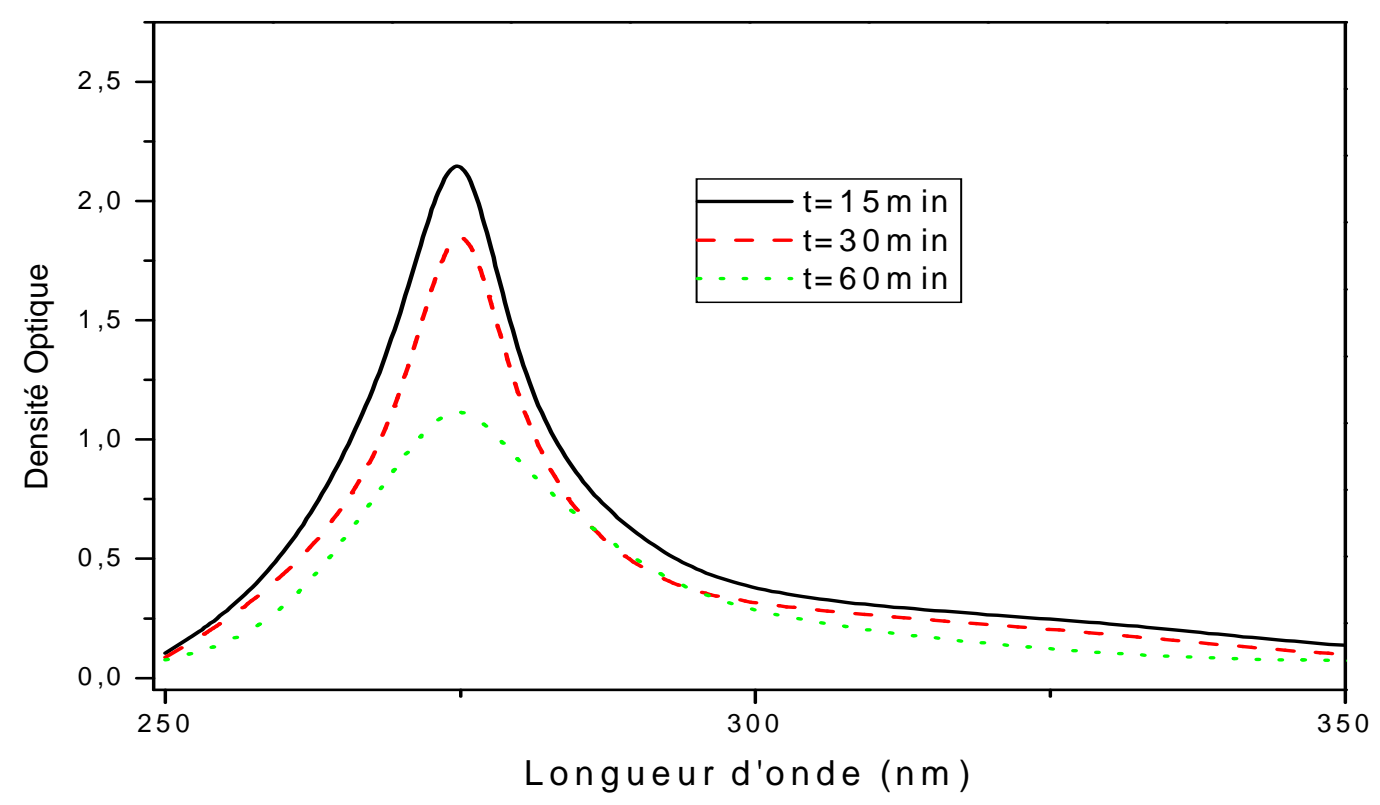

Figure 7: Spectres de la fraction $\mathrm{F}_{2}$ des anthocyanes de Ficus capensis chauffée à $373 \mathrm{~K}$ à différents temps d'exposition. 


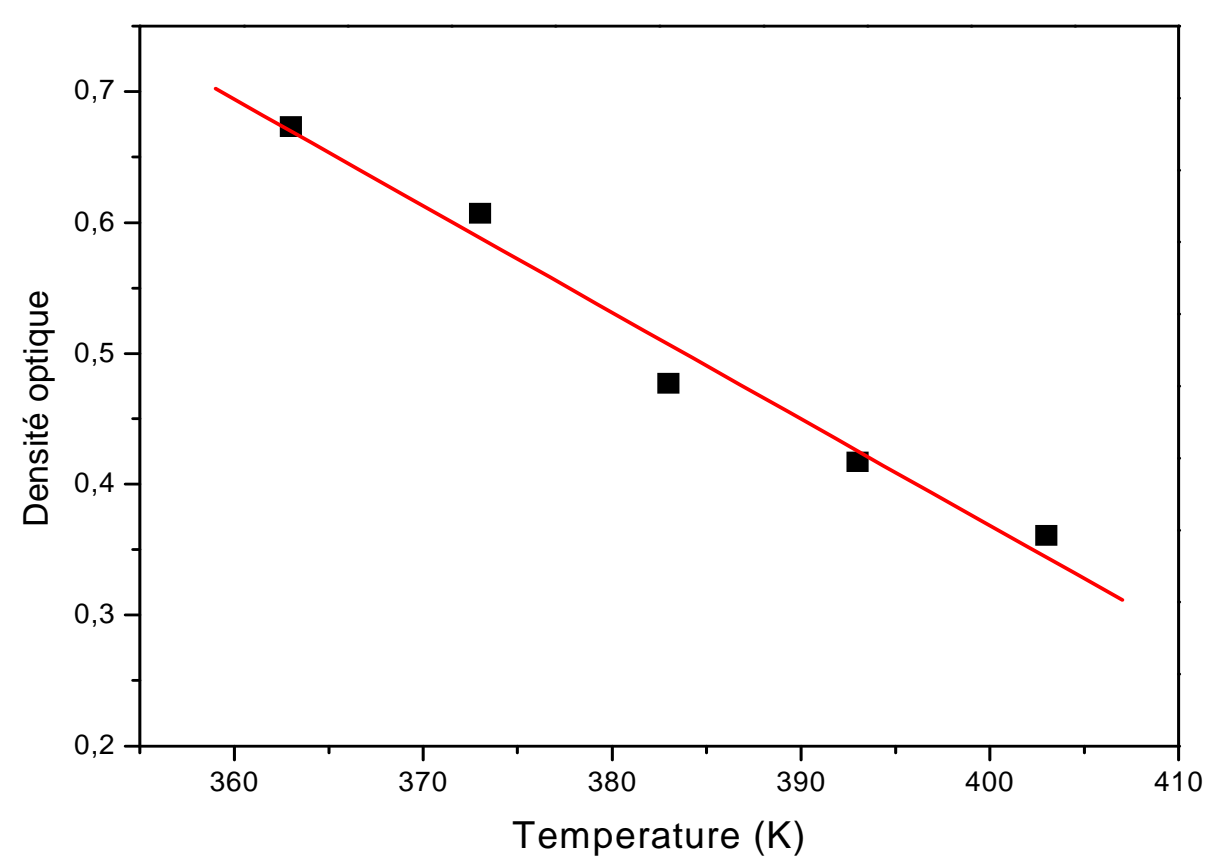

Figure 8 : Variation de la densité optique de la fraction $F_{2}$ d'anthocyanes après exposition à la chaleur à différentes températures pour un temps d'exposition de 60 minutes $(\lambda=275 \mathrm{~nm})$.

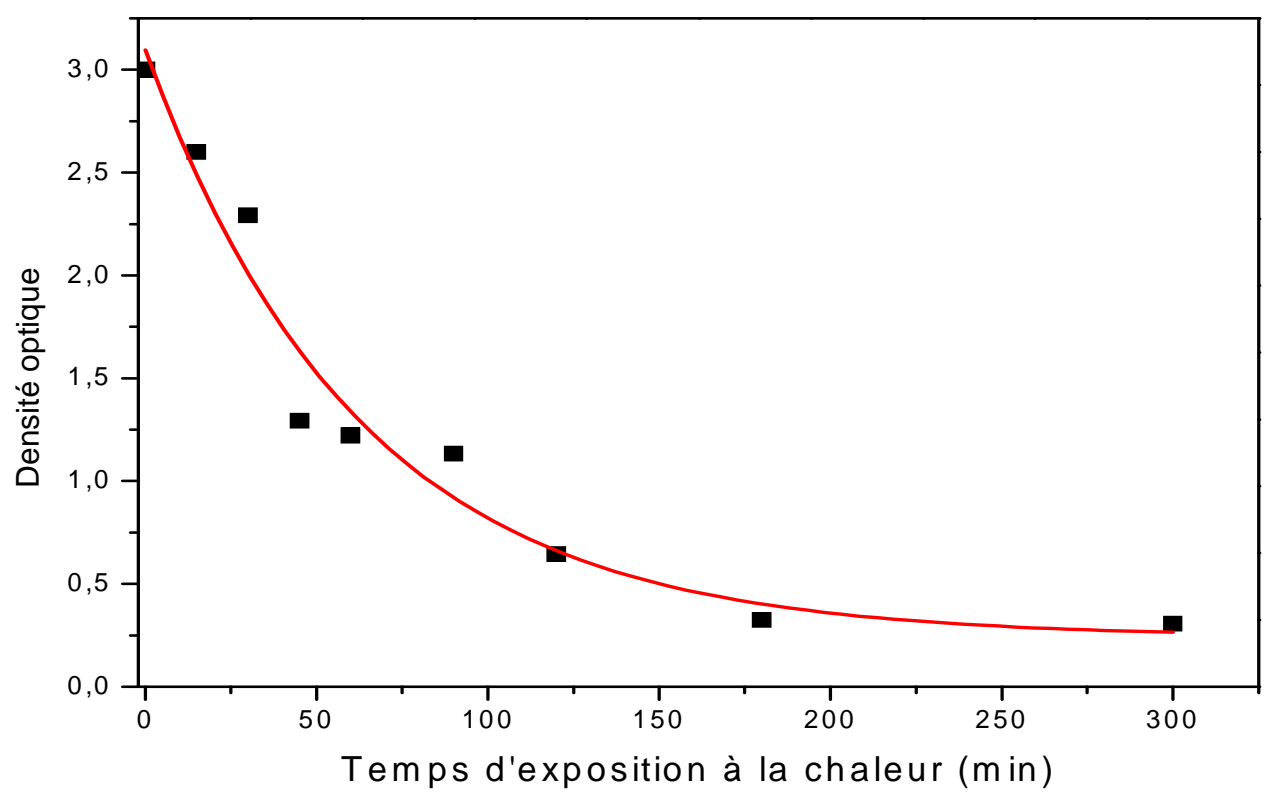

Figure 9 : Evolution de la densité optique de la fraction $\mathrm{F}_{2}$ d'anthocyanes isolée en fonction du temps d'exposition à $363 \mathrm{~K}(\lambda=275 \mathrm{~nm})$.

augmente avec la polarité. Ce qui indique que le groupe chimique responsable de l'activité biologique serait polaire selon le principe «like dissolves like». Etant donné que les anthocyanes sont des substances polaires et que nos résultats antérieurs ( Mpiana et al., 2007b; 2007c; 2007d; Mpiana et al., 2008a; 2008b; 2008c; Mpiana et al., 2009) ont montré 
leur activité antifalcémiante, nous avons testé leur présence dans les quatre extraits. Les résultats obtenus indiquent l'absence de ces composés polyphénoliques dans les extraits moins polaires et leur présence abondante dans les extraits méthanoliques et aqueux de deux plantes. Nous avons alors extraits les anthocyanes de $F$. capensis et de $S$. quinqueloba et testé leur activité antifalcémiante. Les résultas obtenus montrent que ce sont effectivement ces pigments naturels qui sont à la base de l'activité antifalcémiante de ces deux plantes.

En effet, les anthocyanes sont connues comme ayant la capacité d'interagir avec les protéines. Leur possible interaction avec l'hémoglobine $\mathrm{S}$ pourrait entrer en compétition avec la polymérisation de cette hémoglobine et empêcher ainsi la falciformation des drépanocytes. Car la polymérisation est l'un des facteurs déterminants de la falciformation (Mehanna, 2002). Les anthocyanes sont également connus pour leur pouvoir antioxydant, ils pourraient aussi agir sur le rapport $\mathrm{Fe}^{3+} / \mathrm{Fe}^{+2}$ qui est élevé dans les drépanocytes ou sur la stabilité de la membrane des érythrocytes (Mian et al., 1977 ; Kahkoonen et al., 2003).

$$
\text { L'activité antifalcémiante des }
$$
anthocyanes augmente avec la concentration pour atteindre, à partir d'une certaine concentration, une valeur maximale qui reste constante. Cette concentration est appelée concentration minimale de normalisation (CMN), c'est-à-dire la plus petite concentration à laquelle la valeur maximale du taux de normalisation est atteinte. Cette grandeur caractéristique peut permettre de comparer l'activité de deux plantes. Les valeurs de CMN calculées pour les anthocyanes de ces deux plantes sont de 2,5 $\mu \mathrm{g} / \mathrm{ml}$ avec un taux de normalisation de $89 \%$ pour le $F$. capensis et $3,0 \mu \mathrm{g} / \mathrm{ml}$ avec un taux de normalisation de $78 \%$ pour $S$. quinqueloba. Ces résultats montrent que $F$. capensis a une activité antifalcémiante supérieure à celle de S. quinqueloba (Figure 6). Cette valeur est inférieure à celle trouvée pour un bon nombre de plantes utilisées en médecine traditionnelle congolaise mais elle reste néanmoins supérieure à celle des anthocyanes de Alchornea cordifolia qui est de $0,097 \mu \mathrm{g} / \mathrm{ml}$ (Mpiana et al., 2007b; 2007c).
Après fractionnement des anthocyanes, les fractions les plus actives $F_{2}$ et $S_{1}$ ont donné un spectre UV visible avec une bande d'absorption autour de $270 \mathrm{~nm}$ (Figure 7), ce qui confirme qu'il s'agit bien des anthocyanes. Il est, en effet, connu que les anthocyanes présentent généralement une bande dans l'UV entre 270 et $280 \mathrm{~nm}$ et une bande visible entre 465 et $560 \mathrm{~nm}$. Les coefficients d'extinction dans le visible étant en général plus faibles, la bande visible peut ne pas apparaître pour des faibles concentrations en anthocyanes. Ces bandes seraient dues aux transitions $\pi \rightarrow \pi^{*}$ du noyau falvylium qui est le noyau de base des anthocyanes (Kahkoonen et al., 2003; Mpiana et al., 2009).

En médecine traditionnelle congolaise, le mode de préparation le plus utilisé pour les plantes médicinale est la décoction aqueuse. Les anthocyanes sont pourtant connus pour leur instabilité vis-à-vis de la chaleur. C'est pourquoi nous avons étudié l'influence de la chaleur sur la stabilité de la fraction $\mathrm{F}_{2}$. Les figures 7 et 8 confirment le comportement déjà observé pour les anthocyanes des autres plantes utilisées en médecine traditionnelle congolaise (Mpiana et al., 2008b; 2008c; Mpiana et al., 2009). En effet, la baisse de la densité optique, alors que la concentration en anthocyane reste constante, traduit la transformation de l'anthocyane en un autre produit sous l'effet de la chaleur c'est-à-dire la thermodégradation de cette substance naturelle.

Nous pouvons donc nous intéresser à la cinétique de cette thermodégradation. Si nous considérons un modèle où une molécule d'anthocyane se transforme en un ou plusieurs produits de dégradation (équation [1]), nous pouvons étudier la cinétique de thermodégradation de cette molécule. Même si la concentration de la substance n'est pas connue, la structure de la molécule n'étant pas encore élucidée, nous pouvons par spectroscopie UV-visible suivre l'évolution de la densité optique de l'anthocyane en fonction du temps. Cette dernière étant liée par la loi de Lambert-Beer à la concentration (équation [4]). Le « fitting » des résultas expérimentaux avec l'équation [6] montre la compatibilité des points expérimentaux avec la courbe calculée à partir du modèle mathématique basé sur une cinétique d'ordre 1 (Figure 9). 
Les faibles valeurs des constantes de vitesse (k) obtenues montrent que la thermodégradation des anthocyanes est une réaction assez lente. Mais elle augmente avec la température d'exposition comme le montrent les valeurs de k à 363 et $393 \mathrm{~K}$.

\section{Conclusion}

L'activité antifalcémiante de Ficus capensis et Sterculia qinqueloba, deux plantes utilisées en médecine traditionnelle dans la ville de Lubumbashi (RDC) pour soigner l'anémie SS, a été confirmée in vitro. Cette activité serait due aux anthocyanes. Ces pigments naturels étant connus pour leur instabilité vis-à-vis de la chaleur, l'effet de la chaleur a été vérifié. Il ressort de cette étude que, bien que les constantes de vitesse de dégradation thermique soient faibles, il est impérieux lors de l'utilisation de cette plante contre la drépanocytose, de la préserver contre la chaleur. La macération dans l'eau est donc recommandée au lieu de la décoction ou l'infusion.

\section{REMERCIEMENTS}

Nous sommes reconnaissants à la "Third World Academy of Science (TWAS)" pour son financement (Grant no 07077/LDC/CHE/AF/AC UNESCO PR$3240144804)$.

\section{REFERENCES}

Bruneton J. 1999. Pharmacognosie: Phytochimie-Plantes Médicinales. Tec. and Doc. : Paris.

Courtejoie J, Hartaing I. 1992. Laboratoire et Santé. Saint Paul: Kinshasa.

Eliot R, Davies M, Harmse DJ. 2006. Dermatomyositis-like eruption with longterm hydroxyurea. Dermatolog. Treat., 17(1): 56-60.

Fattorusso V, Ritter O. 1994. Vade-Mecum Clinique du Diagnostique au Traitement (13 édn). Masson: Paris.

Ibrahim H, Sani FS, Danladi BH, Ahmadu AA. 2007. Phytochemical and antisickling studies of the leaves of Hymenocardia acida Tul (Euphorbiaceae), Pakistan Journal of Biological Sciences, 10(5): 788-791.

Iyamu EW, Turner EA, Asakura T. 2002. In vitro effects of NIPRISAN (Mix - 0690), a naturally occurring, potent antisickling agent. British Journal of Haematology, 118: $337-343$.

Kahkoonen MP, Heinamaki J, Heinonen M. 2003. Berry anthocyanins: isolation, identification and antioxidant activities. Journal of Agricultural and Food Chemistry, 83: 1403-1411.

Kaplan JC, Delpech M. 1993. Biologie Moléculaire et Médecine ( $2^{\mathrm{e}}$ édn). Flammarion Médecine-Sciences: Paris.

Mehanna AS. 2002. Sickle Cell anaemia and antisickling agents: then and now. Curr. Med. Chem., 8(2): 79-88.

Mian E, Curri SB, Lietti A, Borbardelli E. 1977. Anthocyanosides and the walls of microvessels: further aspects of the mechanism of action of their protective effect in syndromes due to abnormal capillary fragility. Minerva Med., 68: 3565- 3581.

Moody JO, Ojo OO, Omotade OO, Adeyemo AA, Olumese PE, Ogundipe OO. 2003. Anti-sickling potential of a Nigerian herbal formula (Ajawaron $\mathrm{HF}$ ) and the Major plant component (Cissus populnea L. CPK), Phytother. Res., 17: 1173-1176.

Mpiana PT, Tshibangu DST, Shetonde OM, Ngbolwa KN. 2007. In vitro antidrepanocytary activity (anti-sickle cell anemia) of some Congolese plants. Phytomed., 14:192-195.

Mpiana PT, Mudogo V, Tshibangu DST, Shetonde OM, Ngbolua KN, Mbala MB. 2007. In vitro antisickling activity of anthocyanins extract of a Congolese plant: Alchornea cordifilia. M. Arg. J.Med.Sci., 7(7): 1182-1186.

Mpiana PT, Mudogo V, Tshibangu DST, Shetonde OM, Ngbolua KN, Mbala MB. 2007. In vitro antisickling activity of anthocyanins from Ocimum basilicum L. (Lamiacea). Int. J. Pharmacol., 3(4): 371374.

Mpiana PT , Mudogo V, Tshibangu DST, Shetonde OM, Ngbolua KN, Mbala MB. 2007. Antisickling activity of some congolese plants. In Drug Discovery from African Flora. The $12^{\text {th }}$ Symposium of the Natural Product Research Network for Eastern and Central Africa, Hotel Africana, July 22-26. Kampala: Uganda.

Mpiana PT, Mudogo V, Tshibangu DST, Kitwa EK, Kanangila AB, Lumbu JBS, Ngbolua KN, Atibu EK, Kakule MK. 
2008. Antisickling activity of anthocyanins from Bombax pentadrum, Ficus capensis and Ziziphus mucronata: Photodegradation effect. J. Ethnopharmacol., 120: 413-418.

Mpiana PT, Mudogo V, Tshibangu DST, Ngbolua KN, Tshilanda DD, Atibu EK. 2008. Antisickling activity of anthocyanins of Jatropha curcas L. RPMP, Chemistry and Medicinal Value, 25: 101-108.

Mpiana PT, Mudogo V, Ngbolua KN, Tshibangu DST, Atibu EK, Kitwa EK, Kanangila AB. 2008. In vitro antisickling activity of anthocyanins extracts of Vigna unguiculata (L.) Walp. RPMP, Chemistry and Medicinal Value, 25: 91-98.

Mpiana PT, Mudogo V, Kabangu YF, Tshibangu DST, Ngbolua KN, Atibu EK, Mangwala KP, Mbala MB, Makelele
LK, Bokota MT, 2009. Antisickling activity and thermostability of anthocyanins extract from a congolese plant, Hymenocardia acida Tul. (Hymenocardiaceae). Int. J. Pharmacol., 5(1): 65-70.

Neuwinger HD. 2000. African Traditional Medicine. Mepharm Scientific Publisher: Stuttgart.

Sofowora A, 1998. Plantes Médicinales et Médecine Traditionnelle d'Afrique. Karthala: Paris.

Stryer L, Berg JM, Tymoczko JL. 1997. La

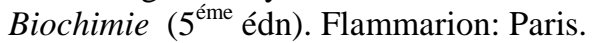

Voet D, Voet JG. 1998. Biochimie ( $2^{\text {nde }}$ édn). De Boeck Université: Paris.

Wajcman H. 2003. Hémoglobine et Hémoglobinopathie, inserm, Paris. http: // www.SickleCelldisease.org. Le 0601 2009. 